\title{
ENRIQUES'S PROJECTIVE GEOMETRY.
}

Vorlesungen über projective Geometrie, von FedERIgo ENRIQUEs, ordentlicher Professor an der Universität Bologna. Deutsche Ausgabe von Dr. Hermann Fleischer, mit einem Einführungswort von Felix Klein. Leipzig, Teubner, 1903. xiv +374 pp., 187 figs. Price 8 marks.

ITAIY has been the home of projective geometry for the last twenty years. In 1894, when Professor Enriques was called to the University of Bologna as professor of projective geometry, he set himself the problem of preparing a systematic treatise on the subject, based on a set of independent axioms. The Lezioni di geometria proiettiva was published at Bologna, in 1898. The next year Hilbert's Foundations of Geometry appeared, at which time Professor Klein expressed the wish for a translation of Enriques's book, in order to have the systematic treatment of projective geometry as well as of metric geometry accessible to German students. The German translation differs from the original only in the correction of various little slips and omissions, the amplification of the appendix, and in the presence of an index. In two respects the make up of the book is hardly up to the Teubner standard; spaced letters are used instead of italics for emphasis, and the figures are rather crude; in other respects the appearance of the book is very presentable.

The axioms, six in number, are the following:

First group, axioms of connection :

I. In a three-dimensional configuration, any two elements determine a one-dimensional configuration to which they belong and which is contained in the given configuration.

II. In a three-dimensional configuration, any three elements not contained in a one-dimensional configuration, determine a two-dimensional configuration to which they belong and which is contained in the given configuration.

III. In a three-dimensional configuration, an element and a configuration of one dimension determine, when the former is not contained in the latter, a two-dimensional configuration to which they belong.

Second group, axioms of order : 
IV. The elements of a configuration of one dimension can be regarded as arranged in a natural cyclic order in either sense.

$V$. If two configurations of one dimension are perspective and an element describes a segment upon one, then the corresponding element will describe a segment on the other.

VI. Dedekind's axiom of continuity.

Numerous illustrations are given to make the meaning of these axioms clear. The advantage of keeping them in the abstract form is seen in their immediate application to dual theorems. The principle of duality is difficult to many students simply because their previous training in analytic geometry has all been on one side. The usual von Staudt construction is given to prove the invariance of the fourth harmonic element, then various metric illustrations are added, each one being marked by a star. This is a decided improvement over von Staudt's arrangement. In the classic Geometrie der Lage all proofs are established by projective principles, but metric illustrations are brought in without warning, which confuses the reader. The present book is, I believe, unique in its direct deduction of each metric theorem as a particular case of a projective theorem. In all other works known to me a few metric properties are established directly, then others are founded on these, no further use being made of projective methods.

The principles thus far established make it possible to prove rigorously the fundamental theorem, after which the idea of the projective group is introduced. This procedure seems for more natural than that employed in the well known books of Reye and of Cremona. The unfortunate place that involution occupies in Reye's book is perhaps the cause of its being frequently slighted by students. In the present work involution comes immediately and naturally after the chapter on projectivity of one-dimensional forms. Chapter 7 enables the author to give a logical purely geometric definition to euclidean geometry. If besides axioms I-VI one assumes the existence of an elliptic involution, having the circular points as double elements, all the theorems of metric geometry can be established. (This is equivalent to having given two right angles with common vertex and common plane.)

Chapter 8 is devoted to projectivity between two forms of two dimensions, with a full discussion of homology and polarity, the latter being defined as follows: a reciprocity (duality) in the plane is a polarity when a triangle exists in which each 
vertex corresponds to the opposite side. Thus far 186 pages have been covered without mentioning any locus or envelope, but nowhere does the presentation seem forced or dry. In particular, the systematic development of each successive topic from axioms $\mathrm{I}$ to VI is rigidly adhered to. Whether students would find this treatment as interesting as that found in most other texts I hardly know, but I believe that those not spoiled by our present excessive instruction in analytic geometry of the point will find it not only profitable but thoroughly enjoyable reading. A conic is now defined as the locus of a point which lies on its polar (or as envelope of a line which passes through its pole), and by means of this definition the usual theory of pole and polar with regard to a conic is easily established. It is readily proved that the conic is also the locus of intersection of corresponding elements of two elementary forms of one dimension. From this standpoint it is evident that the conic is self-dual. The theorems of Pascal and Desargues, together with the usual deductions, are proved and discussed in 12 pages. One objection to this chapter is its rather limited adaptability to problems in construction. A student readily grasps the idea of Steiner's construction and can quickly get a fair idea of the appearance of a locus or an envelope. What the von Staudt method needs is a suitable list of easy exercises and applications.

Chapter 10, projectivity between conics, can be compared with parts of Chapter 11 in Reye's first volume; the present treatment is more general, since it shows that any pair of real elements can be replaced by a suitable pair of conjugate imaginary elements. Added to the usual list of problems of the second degree is a good discussion of Steiner's theorem that every such problem can be solved by means of the ruler and one fixed circle, and the further theorem that all such problems may also be solved by means of a ruler with two parallel edges. Several pages are given to the rigorous discussion of problems of the third degree, one of those treated being the determination of the double elements of two superposed projective spaces of two dimensions. Chapters 12 and 13 are concerned with foci, centers, diameters, and axes of conics. By means of the absolute involution on the infinite line it follows immediately that a point (focus) such that conjugate lines through it are at right angles is necessarily the intersection of isotropic tangents to the conic. The method of proof is everywhere more direct 
than in the older texts, and by means of duality in three dimensions it is shown that many theorems can be applied directly to quadric cones. The chapter on the axes of quadric cones is similar in mode of treatment to the corresponding chapter in Reye.

Finally, the last chapter is devoted to projective spaces of three dimensions. Two proofs are given of the theorem that five pairs of corresponding elenents are sufficient to determine the projectivity. Homology or central (space) perspective, and axial involution are treated at some length. Direct and inverse congruence are shown to be particular cases of homology, the latter being harmonic. An appendix treats of cyclic collineations and the various finite groups defined by them; two pages are given to abstract geometry in which it is shown that no space intuition is needed to develop projective geometry from axioms I to VI; an outline of transformations of space by which spheres are transformed into spheres is followed by a discussion of projective coördinates, imaginary elements and a historical sketch.

It is curious that no mention is made of the hyperboloid except incidentally in connection with the fundamental theorem. In general, with respect to the applicability of the preceding principles to higher configurations, no part of the book is so suggestive as Chapter 11 of Reye, which has furnished the incentive to many a memoir. On the other hand, what one does acquire is a ready confidence in the truth or falsity of a theorem concerning geometric elements. One is not taught to work the machine, but rather to think geometrically. Possibly more time might be required to complete the text than is usually devoted to the subject, but the student who masters it will be better prepared to understand other transformations and the training acquired will be in harmony with his subsequent studies in geometry.

VIRGIL SNYdeR. 